\title{
EDITORIALS
}

\section{Aggression and Violence Among Elderly Patients, a Growing Health Problem}

\author{
Jeffrey L. Jackson, MD MPH and Renee Mallory, MD \\ 'Division of General Internal Medicine, Department of Medicine, Walter Reed Army Medical Center, Washington, DC, USA.
}

J Gen Intern Med 24(10):1167-8

DOI: $10.1007 / \mathrm{s} 11606-009-1099-1$

(C) Society of General Internal Medicine 2009

$\mathrm{V}$ iolence is a pervasive and pernicious poison. Americans are fascinated by violence. US culture is satiated with violence; cartoons average $25-50$ violent acts per hour, ${ }^{1}$ primetime television 8-12 violent acts per hour. Song lyrics are frequently misogynistic and laced with violent themes ${ }^{2}$. Unfortunately, violence is common and takes many welldocumented forms. The US leads the world in homicide rates, particularly among young adults. Like most US health indicators, there is a disparity in violence; the leading cause of death for young Black American men is homicide. Over a quarter of US women have experienced domestic violence in the previous year $^{3}$. Since most victims of domestic violence have children, this form of violence is often witnessed. One in three US women will experience sexual violence at some time in their life ${ }^{4}$. Another emerging, under-reported form of violence is elder abuse, reported to occur in up to $16 \%{ }^{5}$. In this issue of JGIM, Dr. Yamamoto focuses on the health consequences of another common and under reported problem, violence against caretakers by elderly patients ${ }^{6}$.

Aggressive behavior is, unfortunately, a common consequence of dementia. The exact prevalence of violence and aggressive behavior is unknown; there is a wide range of rates in the published literature, partly because of varying definitions and varying, though mostly short durations of patient follow-up. One of the few long-term studies (10 years) found that up to $96 \%$ of patients with dementia demonstrated aggressive behavior over the course of their illness ${ }^{7}$. This is an important problem, particularly as our aging population grows, while long-term health care coverage and the availability of family caregivers shrinks.

How caregivers interpret and report violent behavior is a function of the individual's underlying culture, gender and age. Tensions arise in a caregiver's role, and how they relate to other family members differs among various cultures. Further complicating matters is the dynamism of modern life; families and the lives of their members change over the course of time and across generations. Traditional roles have begun to break down, and most cultures have been experiencing changing social norms. Even in Japan, an extremely paternalistic society with a deep reverence for the aged, Japanese elders fear that the duty of caregiving, traditionally falling on a daughter-in-

Published online September 3, 2009 law, may now be left to a concerned family friend or hired helper.

Violence has been shown to have myriad health effects beyond those sustained directly from the episode. Victims of violence have been shown to have higher rates of depression, anxiety and post-traumatic stress disorder and worse overall health functioning ${ }^{8}$. Children who witness violence in their lives tend to have worse social and health outcomes, with lower cognition and higher rates of somatization. Witnessing violence begets a cycle of violence. Daughters who witness violence in their mother's lives often become involved in violent relationships when they grow up, and sons grow up to become batterers. Violence harms its victims both immediately and also long-term by inflicting deep psychological scars.

That victims of physical and sexual violence experience long-term health consequences is well documented and not surprising. A woman who is battered by her husband or raped on a date often suffers much deeper wounds than the immediate physical effects of the trauma itself. That Dr. Yamamoto found caregivers of aggressive patients reporting higher degrees of caregiver burden is not surprising. This is reflected by the well-known relationship between aggressive behavior and likelihood of eventual institutionalization ${ }^{9}$. Paradoxically, these behaviors often impede placement into nursing facilities, adding to the underlying stress of the caregiver. Of the behavioral and psychiatric symptoms of dementia (hallucinations, incontinence, delusions or depression), caregivers perceive aggressive behavior as the most distressing ${ }^{10}$. Aggression by elderly patients can produce increased stress and increase the possibility of provoking elder abuse of the patient by the caregiver.

Another interesting finding of the study is that caretakers who reported experiencing higher rates of aggressive behavior experienced higher pain scores and worse physical functioning. Indeed, Dr. Yamamoto found a dose-response relationship between violence and deleterious health outcomes. This should not be surprising. The body does not exist independently of the brain. Indeed nearly all the constructs we measure about health are cognitive constructs based on the brain's interaction with reality. Reality itself is a construct created by the brain based on input from the five physical senses. For example, in the case of vision, we don't directly see our environment. Light interacts with cells in the back of our eyes, creating electrochemical stimuli that travel to the brain where it is interpreted. Similarly for hearing, sound waves cause vibrations of the eardrum that are mechanically converted into electrochemical messages that are relayed and interpreted by the brain. Reality is only indirectly experienced. When patients complain that doctors believe their pain is 
"entirely in their head," the reality is that pain is entirely in their head. Nocioceptive receptors trigger electrochemical stimuli that travel to the brain where it is interpreted as pain. Pain may be triggered by a variety of stimuli, but the phenomenon itself is entirely a construct of the brain. Sensory inputs, whether "painful" or "pleasurable," are electrochemical impulses that travel to the brain where they are interpreted and experienced. Dr. Yamamoto's work provides yet another example of the link between "mind and body."

The bottom line for primary care physicians who provide care to elderly demented patients is that they need to be cognizant not only of the health needs of the demented patient, but also of those of the caregiver. Too often caregivers neglect their own health needs and concerns because they are overwhelmed by the needs of their relatives ${ }^{11}$. In the social milieu in which our patients live, we too often neglect the social and environmental context of our patient's lives. Nearly all our patient's health issues are partly individualistic and partly a product of their interactions with their families. Particularly when we provide care to patients with dementia, if we want to be maximally effective, we need to shift our focus from being exclusively on the demented patient to the intricately linked, dynamic pair that is the patient-caregiver dyad.

Corresponding Author: Jeffrey L. Jackson, MD MPH; Division of General Internal Medicine, Department of Medicine, Walter Reed Army Medical Center, Washington, DC, USA (e-mail: Jejackson@usuhs.mil).

\section{REFERENCES}

1. Yokota F, Thompson KM. Violence in G-rated animated films. JAMA 2000;283(20):2716-20.

2. Rich M, Woods ER, Goodman E, Emans SJ, DuRant RH. Aggressors or victims: gender and race in music video violence. Pediatrics. 1998;101(4 Pt 1):669-74.

3. Kramer A, Lorenzon D, Mueller G. Prevalence of intimate partner violence and health implications for women using emergency departments and primary care clinics. Womens Health Issues. 2004; 1(41):19-29.

4. Koss MP. The women's mental health research agenda. Violence against women. Am Psychol. 1990;45(3):374-80.

5. Garre-Olmo J, PLanas-Pujol X, Lopez-Pousa S, Juvinya D, Vila A, Vilalta-Franch J. Prevalence and risk factors of suspected elder abuse subtypes in people aged 75 and older. J Am Geriatr Soc. 2009;57(5):815-822.

6. Yamamoto $\mathbf{Y}$, Hayashino $\mathbf{Y}$, Yamazaki S, Takegami M, Fukuhara S. Violent patient behavior is associated with bodily pain and a high burden on informal caregivers. J Gen Intern Med.

7. Keene J, Hope T, Fairburn CG, et al. Natural history of aggressive behaviour in dementia. Int $J$ Geriatr Psychiary. 1999;14:541-8. doi: 10.1007/s11606-009-1060-3

8. Edwards VJ, Black MC, Dhingra S, McKnight-Eily L, Perry GS. Physical and sexual intimate partner violence and reported serious psychological distress in the 2007 BRFSS. Int J Public Health. 2009; 1 (54 Suppl):37-42.

9. Gilley DW, Bienias JL, Wilson RS, Bennett DA, Beck TL, Evans DA. Influence of behavioral symptoms on rates of institutionalization for persons with Alzheimer's disease. Psychol Med. 2004;34(6): 1129-35.

10. Nagaratnam $\mathbf{N}$, Lewis-Jones $\mathbf{M}$, Scott $\mathbf{D}$, et al. Behavioral and psychiatric manifestations in dementia patients in a community: caregiver burden and outcome. Alzheimer Dis Assoc Disord. 1998;12:330-4.

11. Braun M, Scholz U, Bailey B, Perren S, Hornung R, Martin M. Dementia caregiving in spousal relationships: a dyadic perspective. Aging Ment Health. 2009;13(3):426-36. 